\title{
Teorías de enfermería para la investigación y la práctica en cuidado paliativo*
}

\section{Nursing theories for research and health care practice in palliative care}

\section{Teorias da enfermagem para a pesquisa e a prática em cuidados paliativos}

\author{
Fecha de recepción: 29 de febrero de 2016 \\ Fecha de evaluación: 21 de agosto de 2016
}

Fecha de aceptación: 15 de noviembre de 2016

Publicación en línea: 16 de noviembre de 2016

\author{
Olga Janeth Gómez Ramírez ${ }^{* *}$ \\ Gloria Mabel Carrillo González ${ }^{* * *}$ \\ Edier Mauricio Arias ${ }^{* * * *}$
}

DOI: http://dx.doi.org/10.18359/rlbi.1764

Cómo citar:

Gómez Ramírez, O. J., Carrillo González, G. M. y Arias, E. M. (2016). Teorías de enfermería para la investigación y práctica en cuidado paliativo. Revista Latinoamericana de Bioética, 17(1), 60-79. DOI: http://dx.doi.org/10.18359/ rlbi.1764

\footnotetext{
* Artículo de revisiónn.

* Enfermera, MSc, PhD. Profesora asociada, Facultad de Enfermería, Universidad Nacional de Colombia. Correo electrónico: ojgomezr@unal.edu.co. ORCID: http://orcid.org/0000-0002-9160-4170. Bogotá, Colombia.

*** Enfermera, MSc, PhD. Directora de departamento, Facultad de Enfermería Universidad Nacional de Colombia. Correo electrónico: gmcarrillog@unal.edu.co. ORCID: http://orcid.org/0000-0003-4513104X. Bogotá, Colombia.

*** Enfermero, MSc, Estudiante Doctorado en Enfermería. Becario Colciencias c.727. Correo electrónico: emariasr@unal.edu.co. ORCID: http://orcid.org/0000-0003-2096-1792. Bogotá, Colombia.
} 


\title{
Resumen
}

En este artículo se describen y analizan teorías de enfermería usadas para guiar la investigación y la práctica en cuidado paliativo, por medio de la investigación documental retrospectiva realizada con una ventana de observación de 15 años (1990-2015), y teniendo en cuenta criterios establecidos para los descriptores, teorías de enfermería y cuidado paliativo, que guian la investigación y la práctica en cuidado paliativo. Algunos artículos relacionados y capítulos de libro fueron incluidos como soporte de documentación, y el análisis y la comparación de las teorias fueron guiados a partir de lo propuesto por Fawcett y Desanto (2013).

Se exploraron cuatro teorías de enfermería de rango medio que pueden orientar la investigación y la práctica en cuidado paliativo: confort, final tranquilo de la vida, autotrascendencia e incertidumbre. El análisis de las referencias seleccionadas evidenció el predominio de estudios descriptivos que han abordado los fenómenos centrales de cada teoría en pacientes y en cuidadores familiares. En menor proporción, se identificaron estudios de mayor alcance explicativo sobre variables y aspectos particulares de cuidado paliativo, siendo escasos los que reportan el impacto de intervenciones generadas con base en estos referentes teóricos sobre los pacientes o sus familias. Pudo encontrarse que existen teorías de enfermería relacionadas con cuidado paliativo que requieren mayor exploración y uso en el contexto local, de forma que puedan orientar la investigación en el área y el desarrollo de intervenciones que cualifiquen la práctica con evidencia de impacto sobre los usuarios y sus familias. Palabras clave: cuidados paliativos, teoría de enfermería, literatura de revisión como asunto (DECS).

\begin{abstract}
This article describes and analyzes nursing theories used to guide research and practice in palliative healthcare, through retrospective documentary research conducted with a 15-year observation window (1990-2015), and taking into account established criteria for descriptors, nursing theories and palliative care, guiding research and practice in palliative care. Some related articles and book chapters were included as documentation support, and what Fawcett proposed guided the analysis and comparison of the theories.

We explored four mid-level nursing theories that can guide research and practice in palliative healthcare: comfort, quiet end of life, self-transcendence and uncertainty. The analysis of the selected references evidenced the predominance of descriptive studies that have addressed the central phenomena of each theory in patients and family caregivers. To a lesser extent, we identified studies with a greater explanatory scope on variables and particular aspects of palliative care, few of which report the impact of interventions generated based on these theoretical references on patients or their families. It could be found that there are nursing theories related to palliative care that require greater exploration and use in the local context so that they can guide research in the area and the development of interventions that qualify the practice with evidence of impact on users and their families.

Keywords: palliative care, nursing theory, literature of review as an issue.
\end{abstract}

\section{Resumo}

Neste artigo descrevem-se e analisam-se as teorias de enfermagem utilizadas para orientar a pesquisa e a prática em cuidados paliativos, por meio da pesquisa documental retrospectiva realizada com uma janela de observação de 15 anos (1990-2015), e tendo em conta critérios estabelecidos para os descritores, teorias de enfermagem e cuidados paliativos, que orientam a pesquisa e a prática em cuidados paliativos. Alguns artigos relacionados e capítulos de livros foram incluídos como documentação de apoio, e a análise e a comparação das teorias foram guiadas a partir do proposto por Fawcett.

Exploraram-se quatro teorias de enfermagem nível médio que podem orientar a pesquisa e a prática em cuidados paliativos: conforto, final tranquilo da vida, autotranscendência e incerteza. A análise das referências selecionadas mostrou a predominância de estudos descritivos que tem abordado os principais aspectos de cada teoria em pacientes e em cuidadores familiares. Em menor medida, se identificaram estudos de maior alcance explicativo referente a variáveis e aspectos particulares de cuidados paliativos, sendo escassos os que reportam o impacto das intervenções geradas com base nesses referenciais teóricos sobre os pacientes ou suas famílias. Achara-se que existem teorias de enfermagem relacionadas aos cuidados paliativos que exigem maior exploração e utilização no contexto local, de maneira tal que possam orientar a pesquisa na área e o desenvolvimento de intervenções que qualifiquem a prática com evidência de impacto sobre os usuários e suas famílias.

Palavras-chave: cuidados paliativos, a teoria de enfermagem, literatura da revisão como assunto (Decs). 


\section{Introducción}

Los cuidados paliativos adquieren cada vez mayor importancia dentro de los sistemas de salud. Las demandas de los pacientes y sus familias, así como el interés de los profesionales por evitar el sufrimiento, muestran la progresiva necesidad para la práctica y la investigación en esta área.

La Organización Mundial de la Salud (oms) (2015) establece que los cuidados paliativos consisten en un cuidado total activo del cuerpo, la mente y el espíritu, con la prestación de apoyo a la familia; comienzan cuando se diagnostica la enfermedad. De la misma forma, se sugiere un enfoque multidisciplinario amplio que incluya a la familia y también recursos disponibles en la comunidad, con la participación de un representante de la religión a la que pertenece el paciente y voluntarios, entre otros (Sarmiento, 2011).

Una base conceptual sólida sobre el cuidado paliativo es definitiva para abordar este campo de acción en las profesiones de la salud y demás áreas interesadas. Con el fin de generar dicha fundamentación, se requiere conocer los conceptos y las teorías planteadas en el campo de la salud y el cuidado de enfermería.

Por otro lado, se sabe que una teoría corresponde a un conjunto de conceptos relativamente concretos y específicos que se dirigen a los fenómenos de interés, las proposiciones que describen amplia- mente estos conceptos y las relaciones generales entre dos o más de estos conceptos (Barrera et al., 2008; Fawcett y Desanto, 2013; Sánchez, 2002). Todas estas teorías pueden llegar a tener utilidad en la práctica y esto depende de la satisfacción o beneficio que se obtiene al emplearlas (Sánchez, 2002).

Las teorías varían en su nivel de abstracción y alcance; al tipo más amplio y abstracto de las teorías se denomina como grandes teorías, y al tipo más concreto y específico se le considera teoría de mediano rango. La función de una teoría es documentar de forma concreta un fenómeno; describir y explicar la forma como uno o más conceptos pueden ser evidenciados en la práctica.

Así pues, en el campo de los cuidados paliativos se reconocen propuestas teóricas generales como la de Kubler-Ross (2011) con el modelo fásico del morir, referente mundial en el abordaje de la muerte, personas moribundas y cuidado paliativo. Este modelo plantea las cinco etapas por las que atraviesa la mayoría de pacientes que tienen enfermedades irreversibles, definidas como fases del duelo, que inician con la negación y culminan con la aceptación de la propia muerte, si la resolución es favorable.

Se resaltan además los aportes dados por Saunders (2011) en la evolución de los centros para enfermedades terminales y cuidados paliativos modernos. Saunders fue la primera especialista 
en el manejo de síntomas de pacientes moribundos e inició en Gran Bretaña el movimiento Hospice, considerado un nuevo concepto de abordaje global para enfrentarse a una gran variedad de síntomas y al sufrimiento que experimentan los enfermos terminales (Vidal y Torres, 2006).

Saunders (2011) enfatiza en la importancia de eliminar la angustia terminal y el miedo a padecerla, combinando ciencia sólida y atención personal en los detalles. Indica, además, que toda la familia es la unidad de cuidado, y con la cual se busca compartir cuanta verdad de la situación le sea posible. En el mismo sentido, reconoce la importancia del trabaj o en equipo en los Hospices, compartiendo las propias experiencias de pérdida y cambio, lo que se constituye en una especie de comunidad.

En enfermería existen referentes teóricos que desde una perspectiva humanista y existencialista consideran relevante el cuidado hasta el final de la vida. La enfermería se dirige hacia la comprensión y la intervención en experiencias relacionadas con la salud y el sufrimiento en el que se trabaja con el potencial del ser humano; los participantes en el cuidado desde una perspectiva humanística se involucran en un dialogo intersubjetivo, cuyo fin es fomentar el bienestar en las personas más necesitadas (Snellman et al., 2012).

Los marcos conceptuales anteriormente descritos se constituyen en referentes importantes para cumplir el propósito general de los cuidados paliativos; sin embargo, es necesario avanzar hacia la consideración de teorías rango medio más cercanas a la práctica y la investigación, que puedan ser estudiadas y utilizadas en cuidados paliativos.

Se espera así que las teorías favorezcan una praxis con valor científico, siendo respetuosas de las necesidades de las personas involucradas y de sus particularidades.

El objetivo de este estudio fue describir y analizar teorías de enfermería que pueden guiar la investigación y la práctica en cuidado paliativo.

\section{Método}

Investigación documental retrospectiva con base en la revisión de literatura para dar respuesta a la pregunta: ¿cómo ha sido el desarrollo investigativo de cuatro teorías de enfermería de rango medio para guiar la práctica en cuidado paliativo?

De esta manera, se tuvieron en cuenta los siguientes criterios de inclusión para la selección de las teorías:

- Ser teorías de rango medio de acuerdo con la definición propuesta por Fawcett y Desanto (2013).

- Incluir dentro de sus conceptos fenómenos relacionados con cuidado paliativo. 
- Se contemplaron, posteriormente, los siguientes pasos:

- Análisis de teorias de rango medio de acuerdo con los criterios establecidos por Fawcett y Desanto (2013), que incluyen alcance, contexto y contenido de la teoria. Artículos relacionados y capítulos de libro fueron incluidos como soporte de documentación.

- Búsqueda y análisis de artículos que reportaran el uso de las teorías seleccionadas en la investigación y práctica de cuidado paliativo. Para ello, se realizó una revisión de literatura entre 1990 y 2015 en idiomas inglés y español, bajo los descriptores de teorías de enfermería, cuidado paliativo, confort, final tranquilo de la vida, autotrascendencia e incertidumbre. Se seleccionaron, además, 25 estudios, en los cuales predominan los descriptivos publicados en idioma inglés (tabla 1).

\section{Sintesis integrativa}

A partir de los hallazgos se consolidaron los componentes centrales que han orientado el abordaje del cuidado paliativo como guía para la investigación y la práctica, y así se identificaron las ventajas y las potencialidades que tienen para generar nuevo conocimiento.

\section{Resultados}

\section{Análisis de teorias de rango medio}

En la tabla 2 se presentan los componentes de las teorias seleccionadas, teniendo en cuenta los criterios propuestos por Fawcett y Desanto (2013) para el analisis de teorias.

A continuación, se detallan los principales hallazgos de cada teoría seleccionada

- La teoría de confort se considera un referente fundamental para la práctica del cuidado paliativo. Kolcaba (1994) describe el confort como un estado que experimentan los receptores de las intervenciones. Se reconoce que una de las necesidades centrales de atención de los enfermos en cuidado paliativo y objetivo de la terapéutica es el confort. Visto desde esta perspectiva teórica, implica valorar cuatro contextos: el físico (cualquier comodidad que pertenezca a las sensaciones del propio cuerpo); el psico-espiritual (conciencia interna de este, incluyendo autoestima, autoconcepto, secualidad, significado de la vida); ambiental (factores externos incluyendo el entorno), y el sociocultural (perteneciente a relaciones interpersonales, familiares y sociales) (Kolcaba, 1995, 2001). Una forma de expresar el arte de enfermería es con el cuidado de alivio (Kolcaba, 1995); el confort implica un proceso de acciones reconfortantes y el producto de una mayor comodidad. 
Teorías de enfermería para la investigación y la práctica en cuidado paliativo

Tabla 1. Descripción de artículos seleccionados por teoría

\begin{tabular}{|c|c|c|c|c|}
\hline Teoría & $\begin{array}{c}\text { Términos } \\
\text { de búsqueda }\end{array}$ & $\begin{array}{c}\text { Operadores } \\
\text { booleanos y combinación }\end{array}$ & $\begin{array}{c}\text { Artículos } \\
\text { seleccionados }\end{array}$ & Tipo de artículos \\
\hline $\begin{array}{l}\text { Autotras- } \\
\text { cendencia }\end{array}$ & $\begin{array}{l}\text { Self trans- } \\
\text { cendence, } \\
\text { palliative care }\end{array}$ & $\begin{array}{l}\text { "self"[All Fields]) AND trans- } \\
\text { cendence[All Fields]) AND } \\
\text { ("palliative care"[MeSH Terms] } \\
\text { OR ("palliative"[All Fields] AND } \\
\text { "care"[All Fields]) OR "palliative } \\
\text { care"[All Fields]) }\end{array}$ & 8 & $\begin{array}{l}\text { Cualitativos: análisis de } \\
\text { contenido } 2 \text {. } \\
\text { Cuantitativos: corre- } \\
\text { lacional 3, cuasiexpe- } \\
\text { rimental 2, validación } \\
\text { de instrumento } 1 .\end{array}$ \\
\hline $\begin{array}{l}\text { Final } \\
\text { tranquilo } \\
\text { de la vida. }\end{array}$ & $\begin{array}{l}\text { Peaceful, } \\
\text { end of life, } \\
\text { palliative } \\
\text { care. }\end{array}$ & $\begin{array}{l}\text { ("palliative care"[MeSH Terms] } \\
\text { OR("palliative"[All Fields] AND } \\
\text { "care"[All Fields]) OR "palliative } \\
\text { care"[All Fields]) AND peace- } \\
\text { ful[All Fields] AND (end[A- } \\
\text { ll Fields] AND ("life"[MeSH } \\
\text { Terms] OR "life"[All Fields])). }\end{array}$ & 3 & $\begin{array}{l}\text { Artículos de revi- } \\
\text { sión: } 1 \text {, artículos } \\
\text { cualitativos } 2 \text {. }\end{array}$ \\
\hline Confort. & $\begin{array}{l}\text { Confort and } \\
\text { palliative care. }\end{array}$ & $\begin{array}{l}\text { ("confort")[All Fields]) AND } \\
\text { ("palliative care"[MeSH Terms] } \\
\text { OR("palliative"[All Fields] AND } \\
\text { "care"[All Fields]) OR "palliative } \\
\text { care"[All Fields]). }\end{array}$ & 9 & $\begin{array}{l}\text { Cuantitativo: } \\
\text { validación de } \\
\text { instrumento 2, } \\
\text { descriptivos: } 1 \text {, } \\
\text { cuasiexperimental: } \\
\text { 3, intervención } 1 \text {. }\end{array}$ \\
\hline $\begin{array}{l}\text { Incerti- } \\
\text { dumbre } \\
\text { frente a la } \\
\text { enfer- } \\
\text { medad. }\end{array}$ & $\begin{array}{l}\text { Uncertainty in } \\
\text { illness and pa- } \\
\text { lliative care. }\end{array}$ & $\begin{array}{l}\text { uncertainty"[All Fields]) AND } \\
\text { illness[All Fields]) AND ("pa- } \\
\text { lliative care"[MeSH Terms] OR } \\
\text { " palliative"[All Fields] AND } \\
\text { "care"[All Fields]) OR "palliative } \\
\text { care"[All Fields]). }\end{array}$ & 5 & $\begin{array}{l}\text { Revisión de literatura 2, } \\
\text { cuantitativo } \\
\text { descriptivos 1, } \\
\text { cuasiexperimental, } 1 \\
\text { cualitativo, } \\
\text { fenomenología } 1\end{array}$ \\
\hline Total & & & 25 & \\
\hline
\end{tabular}

Fuente: elaboración propia.

El constructo cuidado de alivio designa una fusión necesaria en la investigación del confort como proceso y producto. Vendlinski y Kolcaba (1997) señalan que el marco teórico de confort es integral y coherente en la evaluación y el diseño de intervenciones para mejorar la comodidad de los pacientes y las familias. El dominio de contenido de comodidad holístico se conceptualiza como partes interrelacionadas (tipos y contextos), ya que se experimentan simultáneamente. 


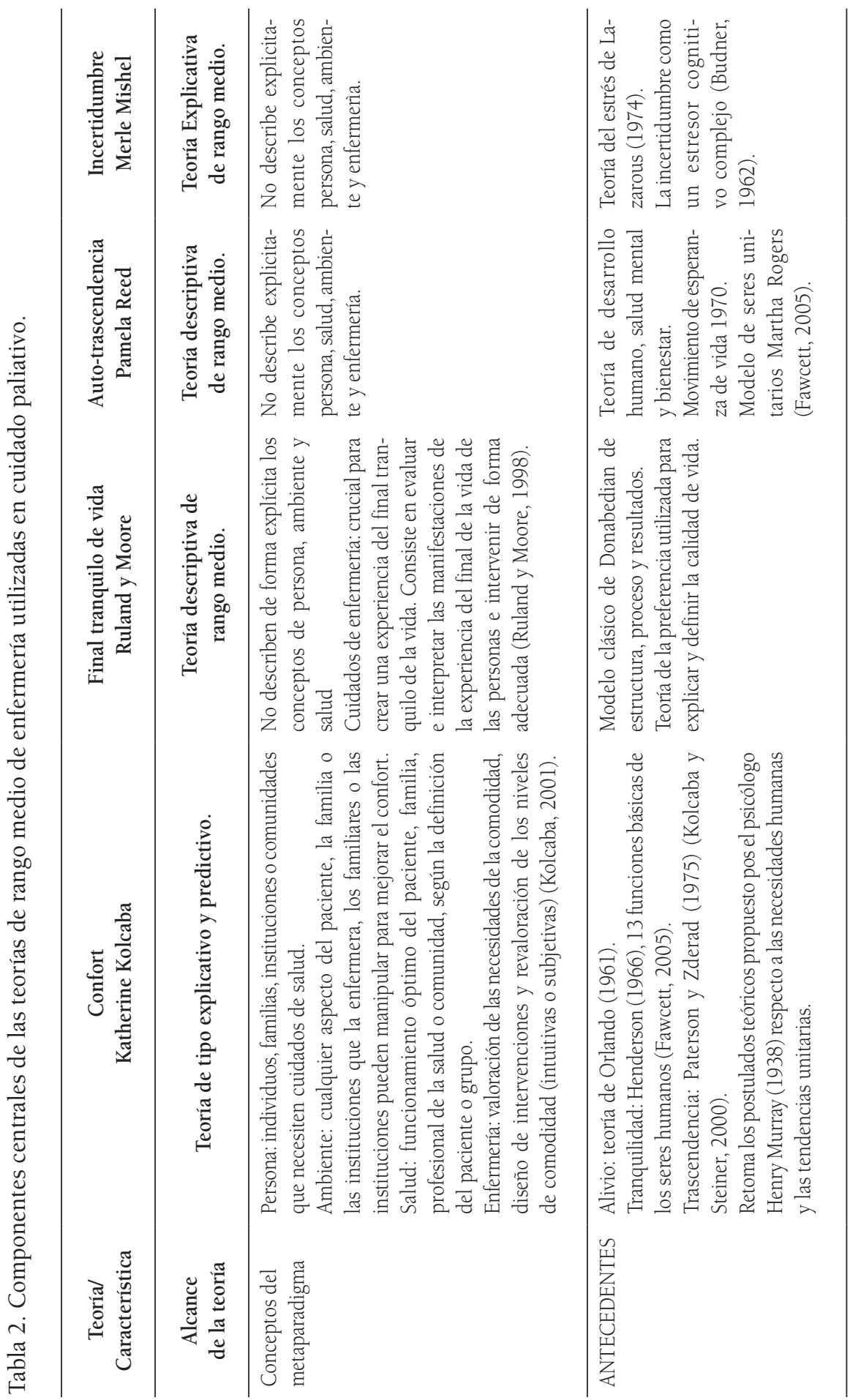




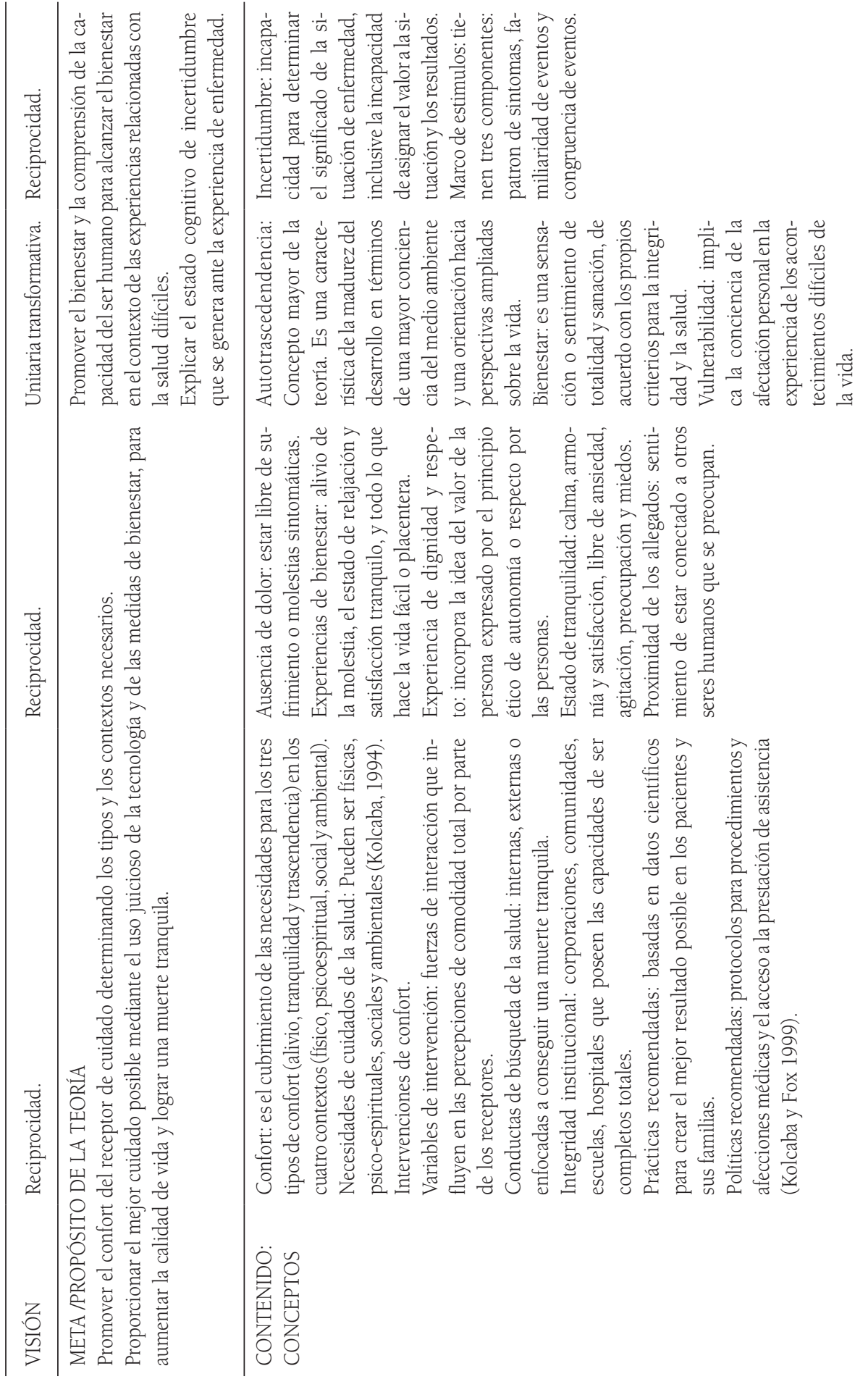




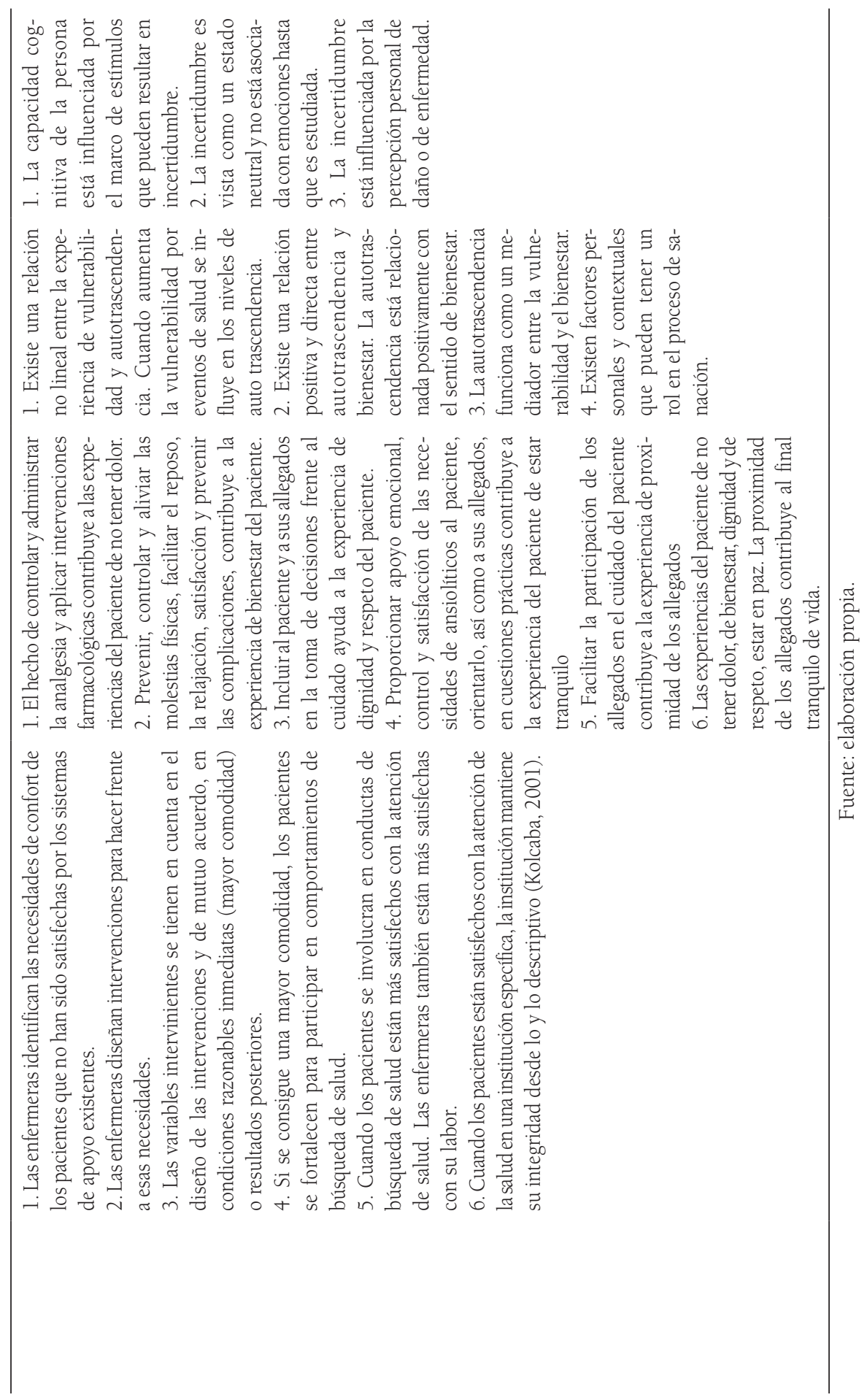


El marco de la atención de confort, que incluye el dominio de contenido y la teoría de la comodidad, se explica y se aplica a través de la presentación de un estudio de caso en un Hospice.

Esta teoría ha sido utilizada como marco de referencia de investigaciones en cuidado paliativo. Al respecto, Novak et al. (2001) midieron los niveles de confort de pacientes en fase avanzada de enfermedad, incluyendo a cuidadores familiares y utilizando el instrumento de confort total, reportando además las propiedades psicométricas de la escala para su uso, tanto en el paciente como en el cuidador. En el mismo sentido, Paiva et al. (2015) realizaron la adaptación transcultural y determinaron las propiedades psicométricas de la versión portuguesa del cuestionario confort holístico-cuidador (HCG-caregiver), en una muestra de cuidadores familiares de pacientes con cáncer en cuidado paliativo; a partir de esto, concluyeron que la escala es válida y confiable para su uso en Brasil.

Hansen et al. (2015) exploraron las relaciones familiares al final de la vida y determinaron las asociaciones entre confort percibido, relaciones y cierre de vida mediante un estudio descriptivo con 30 cuidadores. Señalan, además, que existe una estrecha asociación entre el confort percibido y las interacciones con el cierre de la vida. Sin embargo, indican la necesidad de profundar en estudios sobre las percepciones de las relaciones tanto al paciente como en los miembros de la familia.

Por otro lado, esta teoría ha sido marco orientador de investigaciones que han explorado la eficacia de intervenciones en la práctica. Kolcaba et al. (2004), mediante un ensayo clínico controlado, determinaron la eficacia de los masajes para aumentar el confort y disminuir los síntomas más angustiantes en pacientes cercanos al final de la vida. Dentro de sus hallazgos destacan que los masajes manuales aumentan el confort con el tiempo; no obstante, no evidenciaron cambios en el efecto de estos masajes sobre los trastornos sintomáticos. Por su parte, Magill y Berenson (2008) abordaron los beneficios de la musicoterapia y reflexología como terapias complementarias que pueden calmar y proporcionar comodidad en pacientes al final de la vida, a través de estudios de casos.

Correa y Pereira (2015) verificaron la aplicación de la teoría del confort en las visitas de enfermería realizadas a pacientes en cuidado paliativo con base en la sistematización del cuidado de enfermería, usando taxonomías NANDANIC-NOC. Aplicaron el cuestionario de confort al final de la vida y el noc estado de confort a 20 pacientes en cuidado paliativo en la primera, tercera y sexta visita de la consulta de enfermería. Identificaron, asimismo, tres diagnósticos de enfermería: disposición para mejorar la comunicación, preparación para la toma 
de decisiones mejorada, preparación para la gestión de la salud mejorada, y dos intervenciones de enfermería relacionadas con mayor comodidad para el paciente. Seis diagnósticos de enfermería se asociaron con bajo confort: exceso de volumen de líquidos, fatiga, intolerancia a la actividad, ansiedad ante la muerte, resiliencia deteriorada e impotencia. 12 intervenciones de enfermería se relacionaron con el aumento del confort. Concluyen las autoras la pertinencia de incluir los postulados de la teoría de Kolcaba (1994) en la atención de pacientes que se encuentren en cuidado paliativo.

Brown et al. (2010) estudiaron el impacto de un programa educativo de enfermería sobre el final de la vida para disminuir la ansiedad relacionada con la muerte y el conocimiento del proceso de esta, utilizando los principios de la teoría y práctica propuestos por Kolcaba (1994). Estos autores determinan que la teoría es un referente clave para consolidar estrategias educativas.

- La teoría de autotrascendencia, de Pamela Reed (Reed et al., 2008), plantea entender el bienestar, la trascendencia y la vulnerabilidad del ser humano al final de la vida. En cuidado paliativo, la teoría de autotrascendencia postula que las intervenciones de enfermería fomentan el bienestar y los esfuerzos terapéuticos se dirigen hacia la autoconciencia y la sanidad interior.
En la revisión de literatura acerca de la aplicación de la teoría de autotrascendencia de Reed, en el contexto del cuidado al final de la vida, se aborda el concepto de autotrascendencia desde: 1) la perspectiva personal del participante; 2) los cambios sucedidos a causa de la autotrascendencia; 3) las situaciones desencadenantes de procesos complejos de autotrascendencia, y 4) los factores que la favorecen o no, reconociéndose como un proceso fluido y no como un estado estático u objeto alcanzado.

De forma general, los artículos de abordaje cuantitativo incluyen la autotrascendencia como una variable medible. Iwamoto et al. (2011) determinan el nivel de autotrascendencia entre pacientes con enfermedades incurables y personas de una población sana. Los resultados del estudio muestran que los pacientes que atraviesan eventos importantes o que marcan un antes y un después en su vida aumentan su nivel de autotrascendencia; en este caso, el tener una patología incurable aumenta dicho nivel. Los investigadores comprueban con estos resultados lo planteado en la teoría de Reed, haciendo énfasis en que eventos significativos en la vida como las enfermedades pueden aumentar el nivel de autotrascendencia de la persona. En otro estudio, Norberg et al. (2015) midieron la autotrascendencia en una población de 203 adultos mayores (>85 años), durante cinco años, para describir la asociación entre esta y los eventos negativos en la vida de los participantes. Con esto encontraron que 
a mayor cantidad de eventos negativos en la vida de las personas, se reportan niveles más bajos de autotrascendencia; adicionalmente, identificaron niveles más altos de autotrascendencia después de cinco años de seguimiento a los participantes.

Desde esta misma línea, Kim et al. (2014) diseñaron un modelo que explicara la relación entre la depresión en adultos mayores y su asociación con variables espirituales (autotrascendencia) y propósitos de vida. Dentro de este modelo, incluyeron al cuidador familiar y su influencia en la depresión de los adultos mayores. Los investigadores prueban las tres hipótesis del modelo y establecen que existe una asociación positiva entre la autotrascendencia y el propósito de vida; que el propósito de vida del adulto mayor y del anciano se relacionan positivamente y que existe una relación inversa entre el propósito de vida del anciano y sus síntomas depresivos; sin embargo no evidenciaron asociación entre el propósito de vida del cuidador y la depresión del adulto mayor.

Otros estudios identificados aplicaron intervenciones para aumentar los niveles de autotrascendencia en los participantes, como es el caso de Wasner et al. (2005), quienes evaluaron el efecto que tiene en profesionales de la salud una intervención de entrenamiento en cuidado espiritual. Los resultados documentan que mejora significativamente el nivel de compasión hacia los pacientes y describe la autotrascendencia como una característica inmersa en el cuidado espiritual del paciente, la cual puede ser objeto de intervención. McCarthy et al. (2015), realizaron una intervención piloto para aumentar los niveles de autotrascendencia en adultos mayores. En sus resultados encuentran que, a pesar de existir diferencias entre los grupos muestra y control después de la intervención, estos cambios no son estadísticamente significativos.

En el campo de los artículos cualitativos, los estudios describen desde la perspectiva de los participantes el concepto de autotrascendencia. Mayan (2010) refieren desde la percepción de los pacientes y cuidadores los conceptos de autotrascendencia y autoreformulación; señalan, además, que los participantes al pasar por un momento difícil en su vida experimentan la autoreformulación y autotrascendencia, lo que los lleva a reordenar sus prioridades en la vida, a valorar su experiencia o, como lo describieron los participantes, a valorar su sufrimiento. Hermann (2001) después de realizar 19 entrevistas semiestructuradas a pacientes terminales concluye que la espiritualidad comprende el componente de relación con Dios y una parte de su existencia total. Encuentra asimismo seis necesidades espirituales al final de la vida, que se vinculan estrechamente con el propósito y significado de la vida.

En resumen, los estudios mencionados anteriormente abordan la autotrascendencia desde diversas perspectivas, 
usan la teoría de Reed para realizar sus planteamientos o comparar sus hallazgos. Es importante resaltar que en varias de estas investigaciones se utilizó como instrumento de medición central la escala de autotrascendencia de Reed.

En cuanto a la teoría del final tranquilo de la vida de Ruland y Moore, a diferencia de lo esperado, la revisión de literatura arrojó una baja cantidad de artículos que muestren la aplicación o referencia de esta en personas al final de la vida o con enfermedad avanzada. Esta revisión reportó solo cuatro estudios, dos de los cuales utilizaron un abordaje cualitativo, y los restantes hicieron una revisión de literatura sobre el final tranquilo de la vida y conceptos relacionados con el tema.

Dentro de los abordajes cualitativos se encuentra el estudio de Kongsuwa y Locsin (2009), quienes exploraron la forma como las enfermeras de cuidados intensivos en Tailandia promovían la muerte tranquila de sus pacientes; adicionalmente, se describe desde la perspectiva de la práctica asistencial el concepto de muerte tranquila. En los hallazgos del estudio, se mencionan tres dimensiones que surgen a partir de la información recolectada: conciencia de la muerte, creación de un ambiente de cuidado y promoción del cuidado al final de la vida.

Los autores de la investigación señalan la similitud de las categorías de este estudio con lo propuesto en la teoría del final tranquilo de la vida. Por ejemplo, comparan la creación de un ambiente de cuidado y el promover el cuidado al final de la vida con los conceptos de no sentir dolor, estar cómodo y tener dignidad y respeto. En otro estudio fenomenológico, Kongsuwan et al.(2012) describen los conceptos de la muerte tranquila en unidades de cuidado intensivo desde la perspectiva de familias budistas. Los hallazgos exponen cinco cualidades centrales para el morir tranquilamente en una Ucr: 1) saber que la muerte es inminente; 2) preparación de un estado tranquilo de la mente; 3 ) no sufrimiento; 4) estar acompañado, y 5) familiares sin remordimientos. Cuatro de las cinco cualidades para morir en paz, descritas desde la perspectiva de los familiares, se encuentran en concordancia con lo dicho por diferentes autores a través de diversos contextos, entre ellos Ruland y Moore.

La revisión de Shih-Yi et al. (2009) describen las características de los pacientes en una unidad de cuidados intensivos y determina las principales barreras que no permiten practicar un buen cuidado al final de la vida. Son varias las características que se deben tener para dar un final tranquilo a la vida del paciente, como la autonomía, el cuidado paliativo, la ética y la justicia, que se relacionan estrechamente con las descritas por Ruland y Moore en su teoría. Por otra parte, con un objetivo similar y en el mismo tipo de población, Kirchhoff (2002) describe a través de una revisión cómo un paciente en cuidados 
intensivos, catalogado como terminal, puede tener un buen cuidado al final de la vida. En su revisión la autora establece que la muerte tranquila es sinónimo del buen morir y la describe como aquella que es libre de estrés y sufrimiento para el paciente y la familia, lo que va en concordancia con los deseos del paciente y es consistente con los estándares éticos, clínicos y culturales.

En general, de esta revisión se puede ver cómo el concepto de final tranquilo de la vida ha sido abordado en mayor medida en las unidades de cuidados intensivos, donde la complejidad del cuidado y la gran cantidad de cuidado técnico e instrumental puede ir en contra de lo que es el final tranquilo de la vida. En cuanto a las revisiones de literatura expuestas aquí, se puede ver la necesidad que existe de detallar más claramente lo que es y no es el final tranquilo de la vida y los cuidados relacionados a esta fase. Como conclusión, es importante reflexionar sobre las ventajas que la teoría de Ruland y Moore podría traer en su aplicación a pacientes en cuidados paliativos y al final de la vida.

Por su parte, la teoría de incertidumbre ha sido usada ampliamente como referente para describir la incertidumbre ante la enfermedad y su relación con diversas variables, tanto en los pacientes como en sus cuidadores. De la misma forma, se ha consolidado como una guía para la investigación sobre procesos de adaptación y afrontamiento en enfermedades crónicas y agudas (Reed,
Smith, y Liehr, 2008). Existen instrumentos que miden la percepción de las personas acerca de la incertidumbre ante la enfermedad; sin embargo, son limitados los estudios que se reconocen específicamente en el área de interés, lo que hace llamativo este hallazgo. En general, los autores identifican la incertidumbre con un abordaje psicosocial que comprende un marco de estímulos que pueden desencadenar la vivencia de incertidumbre; en este sentido, el cuidado paliativo es un escenario donde se tienen estímulos negativos y positivos que pueden influir en la incertidumbre $y$, por tanto, requieren ser abordados en la adaptación de la diada persona enferma-cuidador familiar.

En situaciones de enfermedad crónica, los estudios de Gómez et al. (2015) y Bailey et al. (2010) indican que la incertidumbre está influenciada por un conjunto de estímulos que no siempre tienen una connotación negativa. Cuando la incertidumbre es concebida como una oportunidad, las personas avanzan hacia una reestructura cognitiva y en la búsqueda de alternativas para sentirse apoyados afrontando la enfermedad; por el contrario, cuando la incertidumbre se percibe como una condición negativa, en la mayoría de estudios incluidos en el presente artículo, se asocia la incertidumbre con síntomas que afectan a la persona como la fatiga, la depresión, ansiedad, estrés, el dolor. Torrents et al. (2013), a partir de una revisión de literatura, señalan que la incertidumbre puede ser la mayor fuente de ansiedad y 
estrés, aspecto que provoca reactividad fisiológica y aumenta la emocionalidad del paciente. Concluyen las autoras que es importante que las enfermeras desarrollen habilidades para diagnosticar y tratar la incertidumbre con el fin de disminuir su impacto negativo en el estado, psicológico y filosófico. En el mismo sentido, Trejo (2012) indica que la incertidumbre provocada por la enfermedad puede hacer que las personas evalúen inadecuadamente una situación estresante; la aplicación de la teoría de Mishel conduce al profesional en la identificación de los primeros datos de incertidumbre para realizar un plan de cuidados que permitan al paciente y a la familia afrontar la enfermedad.

Lin et al. (2010), mediante un estudio correlacional en Taiwán con una muestra de 205 madres y 96 padres de 226 niños con cáncer, encontraron que aunque la incertidumbre tiene un impacto negativo para los padres, también es una oportunidad de aprendizaje de estrategias de afrontamiento, de mayor interacción con los miembros de la familia y de encontrar significado en la vida.

Desde abordajes cualitativos, se identificó el estudio realizado por Peñaloza et al. (2013) quienes exploraron la percepción de la muerte en personas portadoras del viH en situación de SIDA en etapa terminal. La mayoría de participantes definieron su enfermedad como una maldición que solo se cura con la muerte, y una minoría considera que su condición es una lección de vida. Asimismo, las personas perciben que evitar pensar en la muerte los aleja de ella, los hace reflexionar ante esta y pensar en las cosas materiales y los seres queridos que abandonarán. Concluyen los autores que la incertidumbre frente a la enfermedad y la fase final de esta es un tema central para ser manejado en los pacientes portadores de viH.

En cuanto a estudios de intervención, se reconoció el de Latimer et al. (1998), quienes mediante un ensayo clínico controlado durante un periodo de dos años de seguimiento determinaron eficiencia del Patient Care Travelling Record (PCTR) (herramienta para transmitir información clínica importante acerca del paciente de cuidados paliativos) en el nivel del dolor, el estado de ánimo, la satisfacción con el cuidado, la utilización de los servicios de salud y el nivel de incertidumbre. Los autores señalan la eficacia de esta herramienta en la reducción de la incertidumbre del paciente; sin embargo, plantean el reto que representa este tipo de investigaciones en cuidado paliativo teniendo, en cuenta la alta vulnerabilidad de los participantes, las dificultades de reclutamiento y la alta deserción debido al fallecimiento.

\section{Conclusiones}

El análisis de cuatro teorías de rango medio y su relación con el cuidado paliativo ratifican la importancia de contar con marcos de referencia que orienten la práctica clínica y la investigación en 
enfermería. No obstante, a pesar de lo esperado, se encuentra limitada la producción científica que evidencie el alcance de estas teorías con indicadores de impacto para el paciente o su familia en el campo del cuidado paliativo. Por otro lado, los reportes son en su mayoría de otros contextos, siendo nulos los hallazgos para Colombia.

Se reconoce literatura que invita a la reflexión sobre la oportunidad que representa apropiarse de teorías de enfermería, siendo la mayor parte de esta revisiones o estudios descriptivos. De igual manera, se identifican instrumentos que han sido validados para cuidado paliativo, tanto en pacientes como en cuidadores familiares, aspecto fundamental para consolidar indicadores empíricos que den cuenta de los fenómenos de cuidado propuestos en cada una de las teorías.

Por otro lado, se identifican diversas ventajas de las teorías, ya que reconocen y abordan fenómenos centrales en cuidado paliativo: bienestar y calidad de vida; manejo de síntomas y confort; la vinculación de la familia y allegados y derechos del enfermo, respeto y dignidad. Aportan además en componentes teóricos y conceptuales que facilitan la comprensión de los fenómenos y se constituyen en soporte para argumentar las intervenciones y validar escalas e instrumentos relacionados.

La teoría de confort promueve el confort en el receptor de cuidado, determinando los tipos y los contextos necesarios a saber: físico, psicoespiritual, ambiental y sociocultural. Los estudios identificados revelan el uso de la teoría como base para la validación y adaptación de la escala de confort, la caracterización de necesidades de los enfermos y sus cuidadores la generación de intervenciones de enfermería.

La teoría de autotrascendencia aporta en la comprensión del bienestar, la trascendencia y la vulnerabilidad del ser humano al final de la vida. Se explora el concepto de trascendencia desde la perspectiva del participante, de los cambios sucedidos, de las situaciones desencadenantes y de los factores que la favorecen o no; de esta forma, se reconoce como un proceso fluido y no como un estado estático u objeto alcanzado. Asimismo, algunos estudios ilustran el efecto de intervenciones de enfermería desde esta perspectiva.

La teoría del final tranquilo de la vida de Ruland y Moore expone la necesidad de proporcionar el mejor cuidado posible mediante el uso juicioso de la tecnología y de las medidas de bienestar, para aumentar la calidad de vida y lograr una muerte tranquila, lo que enfatiza en los componentes de ausencia de dolor, experiencias de bienestar, dignidad y respeto, estado de tranquilidad y proximidad de los allegados.

La teoría de incertidumbre permite la comprensión del estado cognitivo de incertidumbre que se genera ante 
la experiencia de enfermedad, es un referente central para estudios descriptivos, explicativos y predictivos en los que se ha explorado el fenómeno.

Finalmente, se considera determinante abordar las teorías de enfermería para su uso en cuidado paliativo desde la docencia a partir de la cualificación del recurso humano en estas y del acercamiento a la práctica clínica con el diseño de intervenciones que evidencien su impacto en el paciente, su familia, allegados y el mismo equipo de salud.

\section{Referencias}

Bailey, D.E., Barroso, J., Muir, A. J., Sloane, R. et al. (2010). Patients with chronic hepatitis $\mathrm{C}$ undergoing watchful waiting: Exploring trajectories of illness uncertainty and fatigue. Research in Nursing E Health, 33(5), 465-473.

Barrera, L., Pinto, N., Sánchez, B. (2008). Hacia la construcción de un modelo de cuidado de cuidadores de personas con enfermedad crónica. Actualizaciones en Enfermería, 11(2), 23 - 29.

Brown, P., Anderson, E.S., Redican, K.J. y Stratton, R. (2010). Studying the effects of the end-of-life nursing education consortium at the institutional level. Journal of Hospice \& Palliative Nursing, 12(3), 184-193.

Stanley Budner, N. Y. (1962). Intolerance of ambiguity as a personality variable. Journal of Personality, 30(1), 29-50.

Correa, L.A. y Pereira, C. (2015). Palliative care in HF: The comfort as an outcome of nursing care. Heart \& Lung: The Journal of Acute and Critical Care, 44(6), 547.

Fawcett, J. (2005). Contemporary nursing knowledge: Analysis and evaluation of nursing models and theories. En The structure of contemporary nursing knowledge (pp. 1-48).

Fawcett, J. y Desanto, S. (2013). Contemporary nursing knowledge: Analysis and evaluation of nursing models and theories (3ra Ed.). Davis Company: Philadelphia.

Gómez, I., Castillo, I., Alvis y Estrada, L. (2015). Incertidumbre en adultos diabéticos tipo 2 a partir de la teoría de Merle Mishel. Aquichan, 15(2), 210218. Recuperado de http://aquichan. unisabana.edu.co/index.php/aquichan/ article/view/3969/html

Hansen, D.M., Higgins, P.A., Warner, C.B. y Mayo, M.M. (2015). Exploring family relationships through associations of comfort, relatedness states, and life closure in hospice patients: a pilot study. Palliative and Supportive Care, 13(02), 305-311.

Hermann, C.P. (2001). Spiritual needs of dying patients: a qualitative study. Oncology Nursing Forum, 28(1), 67-72.

Iwamoto, R., Yamawaki, N. y Sato, T. (2011). Increased self transcendence in patients with intractable diseases. Psychiatry and clinical neurosciences, 65(7), 638-647.

Kim, S.S., Hayward, R.D. y Reed, P.G. (2014). Self-transcendence, spiritual perspective, and sense of purpose in family caregiving relationships: a mediated model of depression symptoms in Ko- 
rean older adults. Aging Mental Health, 18(7), 905-13.

Kirchhoff, K.T. (2002). Promoting a peaceful death in the ICU. Critical Care Nursing Clinics of North America, 14(2), 201-206.

Kolcaba, K.Y. (1994). Una teoría de la comodidad holístico para la enfermería. Journal of Advanced Nursing, 19(6), 1178-1184.

Kolcaba, K. (1995). Confort como proceso y producto, se fusionó en el arte integral de enfermería. Revista de Enfermería Holística, 13(2), 117-131.

Kolcaba, K. y Fox, C. (1999). Los efectos de la imaginación guiada sobre la comodidad de las mujeres con cáncer de mama en estadio precoz de someterse a la terapia de radiación. Foro de Enfermería Oncológica, 26(1), 67-72.

Kolcaba, K. y Steiner, R. (2000). La evidencia empírica de la naturaleza de la comodidad holística. Revista de Enfermería Holistica, 18(1), 46-62.

Kolcaba, K. (2001). Evolución de la teoría de rango medio de la comodidad para la investigación de resultados. Nursing Outlook, 49(2), 86-92.

Kolcaba, K., Dowd, T., Steiner, R. y Mitzel, A. (2004). Efficacy of hand massage for enhancing the comfort of hospice patients. Journal of Hospice \& Palliative Nursing, 6(2), 91-102.

Kongsuwan, W. y Locsin, R.C. (2009). Promoting peaceful death in the intensive care unit in Thailand. Internatinal Nursing Review, 56(1), 116-22.

Kongsuwan, W., Chaipetch, O. y Matchim, Y. (2012) Thai Buddhist families' perspective of a peaceful death in ICUs. Nursing in Critical Care, 17(3), 151-159.

Kubler-Ross, E. y Jáuregui, P. (2011). La muerte: un amanecer. Recuperado de http://www.fundacionnunccoepi.mx/ contenidos/pdf/La \%20muerte\%20 un\%20amanecer.pdf

Latimer, E.J., Crabb, M.R., Roberts, J.G., Ewen, M. y Roberts, J. (1998). The patient care travelling record $\odot$ in palliative care: Effectiveness and efficiency. Journal of Pain and Symptom Management, 16(1), 41-51.

Lazarus, R. S. (1974). Psychological stress and coping in adaptation and illness. International

Journal of Psychiatry in Medicine, 5(4), 321333.

Lin, L., Yeh, C. H. y Mishel, M. H. (2010). Evaluation of a conceptual model based on Mishel's theories of uncertainty in illness in a sample of Taiwanese parents of children with cancer: A cross-sectional questionnaire survey. International Journal of Nursing Studies, 47(12), 1510-1524.

Mayan, M.J., Morse, J.M. y Eldershaw, L.P. (2010). Developing the concept of self-reformulation. International Journal of Qualitative Studies on Health and Well-being, 1(1), 20-26.

McCarthy, V.L., Ling, J., Bowland, S., Hall, L.A. y Connelly, J. (2015). Promoting self-transcendence and well-being in community-dwelling older adults: A pilot study of a psychoeducational intervention. Geriatric Nursing, 36(6), 431-437. 
Magill, L. y Berenson, S. (2008). The conjoint use of music therapy and reflexology with hospitalized advanced stage cancer patients and their families. Palliative and Supportive Care, 6(03), 289-296.

Norberg, A., Lundman, B., Gustafson, Y., Norberg, C. et al. (2015). Self-transcendence (ST) among very old people its associations to social and medical factors and development over five years. Archives of Gerontololy Geriatrics, 61(2), 247-53.

Novak, B., Kolcaba, K., Steiner, R. y Dowd, T. (2001). Measuring comfort in caregivers and patients during late end-of-life care. American Journal of Hospice and Palliative Medicine, 18(3), 170-180.

Organización Mundial de la Salud (oms). (2015). Cuidado paliativos. Recuperado de http://www.who.int/cancer/ palliative/es/

Paiva, B.S.R., de Carvalho, A.L., Kolcaba, K. y Paiva, C.E. (2015). Validation of the Holistic Comfort Questionnaire-caregiver in Portuguese-Brazil in a cohort of informal caregivers of palliative care cancer patients. Supportive Care in Cancer, 23(2), 343-351.

Paterson, J. y Zderad, L., (1975). Humanistic Nursing (2da. Ed.). Nueva York: National League for Nursing.

Peñaloza, M., Mora, M. A., Jaimes, A. Y. y Quintero, A.I. (2013). Percepción de la muerte en personas portadoras del virus de la inmunodeficiencia humana ( $\mathrm{vIH})$ en situación de Síndrome De Inmunodeficiencia Adquirida (SIDA). Revista Ciencia y Cuidado, 10(1), 11-18.

Reed, P. G., Smith, M. J. y Liehr, P. R. (2008). Middle range theory for nur- sing. Nueva York: Springer Publishing Company.

Ruland, C.M., Moore, S.M. (1998). Theory construction based on standards of care: A proposed theory of the peaceful end of life. Nursing Outlook, 46(4), 169-175.

Sánchez, B. (2002). Utilidad de los modelos de enfermería en la práctica. En Grupo de Cuidado, Universidad Nacional (Ed.), El arte y la ciencia del cuidado. (pp. 1330). Bogotá: Unibiblos.

Sarmiento, M.I. (2011). El cuidado paliativo: un recurso para la atención del paciente con enfermedad terminal. Salud Bosque, $1(2), 23-37$.

Saunders, C. (2011). Velad conmigo: inspiración para una vida en cuidados paliativos. Recuperado de http://www.paliativossinfronteras.com/upload/publica/Velad-Conmigo_Cicely-Saunders.pdf

Shih-Yi, L., Chung-Lieh, H., Jun-Hua, L., Shou-Chuan, S. et al. (2009). Attaining Good End-of-Life Care in Intensive Care Units in Taiwan. The Dilemma And the Strategy. International Journal of Gerontology, 3(1), 26-30

Snellman, I., Gustafsson, Christine. y Gustafsson, L.K. (2012). Patients' and Caregivers' Attributes in a Meaningful Care Encounter: Similarities and Notable Differences. ISRN Nursing. 2012, 1-9.

Torrents, R., Ricart, M., Ferreiro, M., López, A. (2013). Ansiedad en los cuidados: una mirada desde el modelo de Mishel. Index de Enfermería, 22(1-2), 60-64.

Trejo, F. (2012). Incertidumbre ante la enfermedad. Aplicación de la teoría para el cuidado enfermero. Enfermería Neurológica, 11(1), 34-38. 
Vendlinski, S. y Kolcaba, K. Y. (1997). Comfort care: A framework for hospice nursing. American Journal of Hospice and Palliative Medicine, 14(6), 271-276.

Vidal, M.A. y Torres, L.M. (2006). In Memorian Cicely Saunders, fundadora de los cuidados paliativos. Revista Socie- dad Española de Dolor, 13(3), 143-144. DOI: $10.5402 / 2012 / 320145$.

Wasner, M., Longaker, C., Fegg, M.J. y Borasio, G.D. (2005). Effects of spiritual care training for palliative care professionals. Palliative Medicine, 19(2), 99-104. 